\title{
Title: Constructing Chinese Lesbian Online Community through \\ Social Media: an Actor-Network Theory-Based Discussion
}

Author: Fei Chen, B.A. ${ }^{1}$; Yuezhou Zhang, $\mathrm{MSc}^{2}$

${ }^{1}$ School of Culture and Communication, Media and Communications, the University of Melbourne, Melbourne, Australia

${ }^{2}$ Department of Biostatistics \& Health Informatics, Institute of Psychiatry, Psychology and Neuroscience, King's College London, London, United Kingdom

Corresponding author

Fei Chen

Email: fc1@student.unimelb.edu.au

Telephone: +86-13020205562

\begin{abstract}
Nowadays, social media plays an essential role in people's life. Compared with heterosexual individuals, social media is more important for lesbian, gay, bisexual, and transgender (LGBT) individuals due to its advantages of anonymity and convenience. Among LGBT individuals, Chinese Lesbians are under greater social pressure due to the influence of traditional Chinese socio-cultural value systems. Building online lesbian communities through social media is one of solutions for helping Chinese lesbians to reduce their stress and protect their rights. However, lesbian social apps have developed notably slowly in a global context. There have been few studies on lesbian apps in the past. The value and utility of lesbian social apps in building an online lesbian community have not been fully explored. This article used "the L-app" (a Chinese lesbian application) as an example and utilized Actor-Network Theory (ANT) to study the values and challenges of the lesbian social app in the establishment of an online
\end{abstract}


community. According to the four foundational stages (problematization, interessement, enrolment, and mobilization) of network construction in ANT, this study discussed the feasibility, values, and challenges of lesbian social apps in building an online lesbian community. Furthermore, this article proposed corresponding potential solutions to these challenges based on ANT. This paper provided support for Chinese Lesbian community constructions in the future.

Key Words: Actor-Network Theory, China, lesbian, Online Community, Social Media

\section{Introduction}

With the development of social networking websites and mobile phone applications (apps), more and more people begin to socialize through social media. Since the digital profile on social media is efficient for face work and impression management (Goffman, 1967, 1978), such as displaying appearance and hobbies, social media provides a means for quickly starting a friendship or relationship between strangers. In addition to finding friends and partners, people can also share their experiences, opinions, and insights on some topics through social media. Therefore, social media plays an essential role in the society nowadays.

Compared with heterosexual individuals, social media is more important for lesbian, gay, bisexual, and transgender (LGBT) individuals (Dhoest, 2016). Since LGBT individuals are suffering from social stigma and discrimination in China, they are more inclined to anonymously express their sexual orientation and find friends and supports on the internet through social media (Chong et al., 2015). Among the LGBT individuals, lesbians in China are under greater social pressure due to the influence of traditional Chinese socio-cultural value systems, such as the Three Obediences and Four Virtues in Confucianism (Chan, 2003; Teng, 1996). Besides, some rights of lesbians are not the same as those of heterosexual women, so their rights cannot be fully protected by the feminist movement. Therefore, Chinese lesbians need a channel to reduce stress, get 
supports, and protect their rights (Choy, 2018). Establishing online lesbian communities through social media (social apps) could be one of the solutions (Harper et al., 2009; Mehra et al., 2004). However, lesbian social apps have developed notably slowly in global context (Murray \& Sapnar Ankerson, 2016). There are few studies on lesbian apps in the past, and the value and utility of lesbian social app in building an online lesbian community have not been fully explored. To fill in this gap, this paper aimed to explore the utility of the lesbian social app in establishing an online lesbian community, as well as the challenges that may be encountered in the process.

Since the interactions and relationships between humans (lesbians) and technology (social apps) in a relation network were analyzed in this study, the actor-network theory (ANT) is a suitable method (Latoru \& Callon, 1981), which highlights the interactions between human and non-human actors (Law, 1992). According to the four foundational stages (problematization, interessement, enrolment, and mobilization) of network construction in ANT (Callon, 1984), we respectively discussed the feasibility, values, and challenges of lesbian social apps in building an online lesbian community. We also proposed corresponding potential solutions to these challenges based on ANT. To the best of our knowledge, this paper is the first attempt to study the value and challenge of the lesbian social app in the establishment of an online lesbian community through ANT.

The structure of this paper is as follows. We first did a literature review, including the history of homosexuality and lesbian in China, the lesbian community, and previous studies related to lesbian social apps. Then, we introduced the main concepts and the core process ('translation') of ANT. Finally, we discussed the values and challenges of the lesbian social app in building an online lesbian community based on four essential processes of translation of ANT.

\section{Literature review}




\section{Homosexuality and Lesbian in China}

Although homosexuality was decriminalized in China in 1997, Chinese LGBT individuals are still suffering from social stigma and have not received equal rights compared with Western countries (Chong et al., 2015). Influenced by the traditional Chinese value system, such as Confucianism and Taoism (Rofel, 2007), same-sex behaviors and partnerships were never fully accepted historically and were regarded as abnormal phenomenon by some heterosexual supporters in China (Burger, 2012). So far, same-sex marriage has not been recognized by Chinese law, and the Chinese government conducts strict media policies and restrictions on disseminating gay-related content, including censoring all LGBT-related issues both online and offline (Mountford, 2010). However, several LGBT groups have begun discussing feminism since 2012 because they lack gender equality within the LGBT community (Cheng, 2018). Gay men receive more attention in society, while lesbianism is little-known and deeply hidden (Engebretsen, 2013). (Kam, 2013) observes that the Chinese Lesbian community is little understood, as lesbian is often marginalized in public discussion and is rarely reflected in the mainstream media. Therefore, in terms of lesbians face two common marginalization phenomena, namely 1) In patriarchal societies, since women are oppressed by gender inequality, they are in a disadvantaged position, marginalized in society (Yip, 2006); 2) In the LGBT community, scholars focus more on gay men (Kam, 2013), further marginalizing lesbians. As a result, this double marginalization presents a distinct challenge to lesbians (Cheng, 2019). Therefore, lesbians are under multiple social pressures and need a channel to seek supports and opportunities to release their desires of rights and sexual orientation. Therefore, the online lesbian community is an important place for China's lesbians to look for gender identity, supports and building relationships.

\section{Lesbian community and lesbian social apps in China}

As early as the 1990 s, lesbian communities in China began to emerge, especially in 
large cities (Engebretsen, 2013). Pew Research Center (2013) indicates that homosexuals face discrimination in many countries. Direct or indirect experiences of being denigrated and shamed as sexual minorities in the family (Ryan et al., 2010). The school, workplace, and society at large prevent some lesbians from forming meaningful interpersonal connections, potentially increasing their risk of engaging in maladaptive coping strategies (Meyer, 2003). As a result, lesbians take several measures to combat stigma, including community building, which allows people to support one another, feel belonging, and develop a sense of commonality (Frost \& Meyer, 2012). Especially in regions where discrimination against sexual minorities is limited, it may be pertinent to recognize and support sexual minorities. Research on the development of gay identities shows that they use digital media frequently than their peers (Gudelunas, 2012). However, lesbian social apps have developed much slower than other social apps (Murray \& Sapnar Ankerson, 2016; Tang, 2017). So far, it remains unclear whether social media is effective for Chinese lesbians. To reach the study's objective of exploring how social media as a tool for lesbian community, the L-app will be a case study (see Fig. 1 The L application screenshots). The L-app is a popular lesbian social networking app with more than 6 million users, originally called "Rela" in China (Google Play, 2020). Aside from (Li, 2020) study of what Chinese queer women living in Australia do under the Rela' live streaming feature, no scholars have examined how lesbians in China use the L-app for social interaction and develop their identity after it officially changed the name and updated more app features. As a result, this study focuses on investigating the interactions between lesbians and the L-app, and the values and challenges of the L app in building the online lesbian community
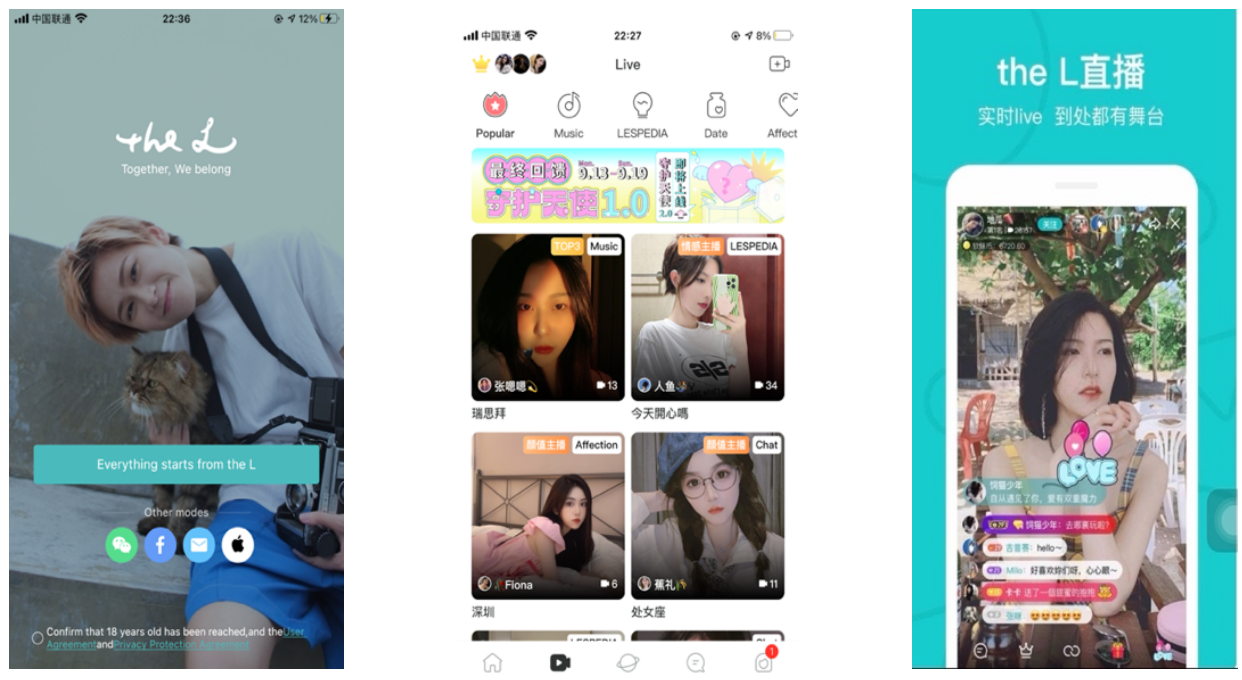
Figure 1. The L application screenshots

\section{Methodology}

\section{Actor-network theory}

Actor-network theory (ANT) was used in this paper to explore the interactions between lesbians and social media and the importance of social media for establishing online lesbian communities. ANT is a social theory proposed by Bruno Latour and Michel Callon in the mid-1980s (Latoru \& Callon, 1981). ANT holds that everything in the social and natural worlds exists in a constantly shifting network of relationships, and there are no external social forces beyond the interactions between the participants in the network. ANT is used to solve some social problems by analyzing the interactions and coordination between participants in the relationship network. The participants in the actor-network are considered as actors, and actors can be both human and nonhuman (such as technology). Human and non-human actors are considered equally important in the ANT; therefore, ANT was applied in different research fields which need to explore the relationships and the interactions between human and non-human actors.

The core of the ANT is the 'translation' process, which is the key to the establishment and stability of a new actor-network. The translation process contains 4 foundational stages: problematization, interessement, enrolment, and mobilization (Callon, 1984). We explained below how these 4 stages were applied to our research question about the construction of an online lesbian community.

1) Problematization: This stage is used to define the purpose of establishing the network and determine one or more core actors. The core actors need to negotiate and set an 'obligatory point of passage' (OPP), which represents the interests and purposes of all actors, to make sure all core actors are willing to join and enact this 
network. In this paper, the original purpose of the establishment of the network is to build a harmonious and stable online lesbian community to protect the rights of lesbians in China. As this paper is an attempt to assess the feasibility of ANT in this field, we simply consider three actors: the lesbian, the L app, and lesbianrelated policy (such as censorships). Among them, the lesbian is the human actor, and the L app and policy are non-human actors. In this stage, we analyzed and discussed the OPP and the interests of both lesbians and the L app.

2) Interessement: Actors may already be in an existing network. In the interessement stage, it is necessary to consider how to dissociate actors from their previous network to join this emerging new network. In this study, lesbians may have their previous ways of looking for friends and supports, such as through offline local lesbian communities or common social apps (WeChat). In this stage, we analyzed how to attract lesbians to choose to use the L app to join the new online lesbian community. We analyzed the advantages of using the L app compared with the other social communication methods.

3) Enrolment: In this stage, actors need to be assigned to different roles in the new actor-network. Their roles are defined by aligned the interests of the network in the problematization stage. In this stage, we discussed the roles of lesbians and the $\mathrm{L}$ app in establishing the online lesbian community.

4) Mobilization: A network may have a few actors as spokespersons. However, when controversies occur, these spokespersons usually tend to protect their own interests in priority. Therefore, this stage is used to confirm the appropriate spokespersons for this network to ensure the interests of most actors are not betrayed. In this stage, we analyzed how lesbians can become one of the spokespersons of the network for the protection of lesbians' rights and interests in the new network.

\section{Discussion}




\section{1) The problematization}

The problematization is the first step to establishing a network. The primary purpose of this step is to clarify the key actors in the network, the goals of the network construction, define all roles and functions of actors, and propose solutions and measures for each actor to accept and recognize mandatory access points ultimately achieve organizational objectives.

Developing gay virtual communities in China is an inevitable development trend based on China's social, cultural, and political background. Although China has not yet clarified its guiding principles and attitudes towards online communities that deal with homosexuality, these kinds of social issues will be lessened the minute the interests of sexual minorities are safeguarded. In that sense, government departments can play a more significant role as instructors, while L-app plays a role as facilitator and lesbians as users. Therefore, there are corresponding interests and obstacles for each player in the lesbian community created by L-app. It is critical for each actor in the L-app network to identify with the L-app's mandatory access point, ensure lesbian social rights, and create a secure online space to achieve the full desired effect.

a) For government: In general, gay social platforms benefit the government by helping to promote same-sex physical health and prevent AIDS. Therefore, if the L-app aids the government in promoting lesbian-related physical health knowledge, the government will support it instead of banning its operations.

b) For the L-app: In China, lesbians rarely attend their families, workplaces, or social gatherings, so they need to establish relationships and networks using other communication channels. Social media can also enable and enhance the identification of support networks they share a sense of belonging. The L-app company enables it to occupy the lesbian app market and generate considerable 
revenue. A majority of the L's revenue comes from membership fees and advertising. Nevertheless, one of the problems with the L-app is that it cannot be publicly and directly promoted like other heterosexual social software because of China's social environment. Consequently, this difficulty has also influenced the company's revenue, making it hard to drive faster and better app development and optimize the app's functionality.

c) For lesbians: According to research in social psychology, individuals have different motivations for joining groups. A person's social identity is derived from the social group's values, attitudes, and behavior they desire to belong to (Hogg \& Hains, 1996) and is shaped by them. For lesbians, it is essential to have support and companionship to obtain and maintain good mental health, which is a massive factor in attaining or maintaining good mental health. There is a good chance queer women scattered all over can virtually connect through the L. Using this space, Chinese queer women can communicate virtually with the L community and build friendships with same-sex couples and friends in the same community. Groups such as these provide emotional support, friendships, and a sense of belonging.

\section{2) The interessement}

As a person's decision to come out is the first step towards becoming self-aware, and their acquaintances' acceptance of their sexual orientation defines the level of intimacy. In China, however, social networking sites such as WeChat have many family members, classmates, colleagues, and so on, and lesbian users would not want their same-sex orientation revealed. As a result, they are unable to express their feelings on such a social media platform. In the same context, several dating apps in China cater to heterosexual dating needs, including Tantan and Soul. The matching software automatically matches profiles of heterosexuals. However, it is harder for the homosexual to find matches based on their preferences, which often causes issues in their dating and dating process. It relates to the cultural background of traditional 
Chinese people, which can be thought of as the non-human actor in ANT. As a result of Confucian filial piety, homosexuality is commonly seen as a source of shame for individuals and their families (Chow \& Cheng, 2010). As families often struggle with issues related to sexual orientation, gay Chinese might find that close friends and other non-relative groups (except for professionals) are particularly valuable. There is no obvious offline lesbian community in China, and lesbians rarely attend their families, workplaces, or social gatherings, so they need to establish relationships and networks using other channels of communication. Using social media, they can establish and maintain social connections efficiently (Bryson, 2004).

In this context, the L-app is an application that creates a queer space for lesbians to connect. For example, by using the "people nearby" function in the L-app, users can tell if the person is near is lesbian. A gay contact's personal data and spatial distance can be viewed on the screen if connected to the app, and the closest is ranked highest. In this way, lesbians could "discover" another previously unknown user of the $\mathrm{L}$ everywhere. Therefore, the L-app as a non-human actor can also enable and enhance the identification of support networks, they share a sense of belonging through. To cope with public scrutiny, many lesbians undertake social interactions online. Online friendships are easier to establish through social networks (Gudelunas, 2012). It is noteworthy that online social networks are usually larger than offline social networks, indicating that online users at least have better self-esteem than offline users. In other words, it reflects their desire for appearance normalization (the desire for "normal" and "a good life"). This is the connection between Actors and Network formed among lesbians on social media. Each of these interacts, restricts, and influences the other.

\section{3) The enrolment}

Creating a social network for lesbians in China involves actors from various perspectives, including L-app users, lesbian promotion channels, and government policies. Each actor has its interests and operational capacity, while their relationship 
influences their behaviour and access to resources.

A key actor in building this lesbian virtual space is the L-app. The L-app's technology developers must understand the social needs of lesbians and establish rules for the app's operation and use so that the virtual community can function correctly. Additionally, the L-app's operational content must comply with the Chinese government's digital control policy regarding gay content to avoid conflicts that can make the app inoperable. Further, the L-app's advertising department should utilize the app through various media channels to promote lesbian awareness of the app and attract new users. Furthermore, while using the L-app, lesbian users also have responsibility for the virtual community, thereby creating a good environment for the lesbian community. Because throughout the L community, there has been some ill-intentioned deception and lies about money. Furthermore, the L needs capital to run its operations, so they must advertise. Because the $\mathrm{L}$ is a lesbian platform, most users will use it privately, which results in less effective advertising, so the platform's advertising costs are not very profitable. For example, related live broadcast content is declining, and the interactive interface is less attractive. Thus, the platform may lose many lesbian users once a better mobile social platform becomes available for Chinese lesbians.

\section{4) The mobilization}

A key component of ANT is mobilization, which is meant to protect the interests of up to a few actors. In the process, the L network serves as an appropriate focal point for lesbian users to be a gathering place and talking point for external voices against lesbian rights, thereby preventing overwhelming state repression. \#IamGay is a classic example of the Chinese LGBT community gaining digital publicity using the hashtag. On April 13, 2018, Weibo announced that it would launch a three-month campaign to remove content that was deemed illegal, such as pornography, violence, and gay-related topics (Liao, 2019). Immediately following the news, many Weibo users called for a boycott on the platform. At that time, lesbians' energy gathering on the L-app provided an 
excellent platform from which they could make a good advocating for a unified coping style. Then, they can cross the platform to Weibo to resist. That is what the L-app does for lesbians when it comes to protecting their interests.

\section{Conclusion}

The L-app is a "Network" that links all types of Chinese lesbians. In the L community, lesbians enjoy feeling closely connected, which creates a solid emotional bond between them. It does not matter where community members live, and they can be very close in spirit. Having this kind of experience can have positive effects such as a sense of belonging and connection, happiness and self-esteem, and the ability to transfer positive effects to others. Additionally, it demonstrates that the L is a crucial social network platform and that the Actor-Network Theory has been more concretely applied in this application. In other words, social media can serve as a resource for Chinese lesbians to cultivate resilience, support one another, combat discrimination, and promote the healthy development of their community. Even though this sample may not accurately represent the diversity of lesbians involved in social networks and media, the construction of online communities is a haven for lesbians and creates a social space.

\section{References}

Bryson, M. (2004). When Jill jacks in: Queer women and the Net. Feminist Media Studies, 4(3), 239-254.

Burger, R. (2012). Behind the red door: Sex in China. Earnshaw Books.

Callon, M. (1984). Some elements of a sociology of translation: domestication of the scallops and the fishermen of St Brieuc Bay. The sociological review, 32(1_suppl), 196-233.

Chan, S. Y. (2003). The Confucian conception of gender in the twenty-first century. Confucianism for the modern world, 312-333.

Cheng, F.-T. (2018). Theorizing TL esthetics: Forming a femme gaze through yes or no 
2.5. Journal of lesbian studies, 25(1), 71-87.

Cheng, F.-T. (2019). Theorizing TL esthetics: Forming a femme gaze through yes or no 2.5. Journal of lesbian studies, 25(1), 71-87.

Chong, E. S., Zhang, Y., Mak, W. W., \& Pang, I. H. (2015). Social media as social capital of LGB individuals in Hong Kong: Its relations with group membership, stigma, and mental well-being. American journal of community psychology, $55(1), 228-238$.

Chow, P. K.-Y., \& Cheng, S.-T. (2010). Shame, internalized heterosexism, lesbian identity, and coming out to others: A comparative study of lesbians in mainland China and Hong Kong. Journal of Counseling Psychology, 57(1), 92.

Choy, C. H. Y. (2018). Smartphone apps as cosituated closets: A lesbian app, public/private spaces, mobile intimacy, and collapsing contexts. Mobile Media \& Communication, 6(1), 88-107.

Dhoest, A. (2016). Feeling (dis) connected: Diasporic LGBTQs and digital media. International Journal of E-Politics (IJEP), 7(3), 35-48.

Engebretsen, E. L. (2013). Queer women in urban China: An ethnography. Routledge.

Frost, D. M., \& Meyer, I. H. (2012). Measuring community connectedness among diverse sexual minority populations. Journal of sex research, 49(1), 36-49.

Goffman, E. (1967). Interaction ritual: Essays on face-to-face interaction.

Goffman, E. (1978). The presentation of self in everyday life (Vol. 21). Harmondsworth London.

Gudelunas, D. (2012). There's an app for that: The uses and gratifications of online social networks for gay men. Sexuality \& Culture, 16(4), 347-365.

Harper, G. W., Bruce, D., Serrano, P., \& Jamil, O. B. (2009). The role of the Internet in the sexual identity development of gay and bisexual male adolescents. The story of sexual identity: Narrative perspectives on the gay and lesbian life course, 297-326.

Hogg, M. A., \& Hains, S. C. (1996). Intergroup relations and group solidarity: Effects of group identification and social beliefs on depersonalized attraction. Journal of Personality and Social Psychology, 70(2), 295. 
Kam, L. Y. L. (2013). Shanghai lalas: Female tongzhi communities and politics in urban China (Vol. 1). Hong Kong University Press.

Latoru, B., \& Callon, M. (1981). Unscrewing the Big Leviathan: How Actors Macrostructure Reality and How Sociologists Help Them Do It. Knorr-Cetina, K. aren; Cicourel, Aaron V.(Eds.). Advances in Social Theory and Methodology: Toward an Integration of Micro-and Macro-Sociologies. Boston: Routledge.

Law, J. (1992). Notes on the theory of the actor-network: Ordering, strategy, and heterogeneity. Systems practice, 5(4), 379-393.

Li, H. (2020). Transnational togetherness through Rela: Chinese queer women's practices for maintaining ties with the homeland. International Journal of Cultural Studies, 23(5), 692-708.

Liao, S. (2019). “\# IAmGay\# What About You?”: Storytelling, Discursive Politics, and the Affective Dimension of Social Media Activism against Censorship in China. International Journal of Communication, 13, 21.

Mehra, B., Merkel, C., \& Bishop, A. P. (2004). The internet for empowerment of minority and marginalized users. New media \& society, 6(6), 781-802.

Meyer, I. H. (2003). Prejudice, social stress, and mental health in lesbian, gay, and bisexual populations: conceptual issues and research evidence. Psychological bulletin, 129(5), 674.

Mountford, T. (2010). The legal status and position of lesbian, gay, bisexual and transgender people in the People's Republic of China. International Gay and Lesbian Human Rights Commission.

Murray, S., \& Sapnar Ankerson, M. (2016). Lez takes time: Designing lesbian contact in geosocial networking apps. Critical Studies in Media Communication, 33(1), 53-69.

Pew Research Center. (2013). The global divide on homosexuality. http://www.pewglobal.org/files/2013/06/Pew-Global-AttitudesHomosexualityReport-FINAL-JUNE-4-2013.pdf.

Rofel, L. (2007). Desiring China. Duke University Press.

Ryan, C., Russell, S. T., Huebner, D., Diaz, R., \& Sanchez, J. (2010). Family acceptance 
in adolescence and the health of LGBT young adults. Journal of child and adolescent psychiatric nursing, 23(4), 205-213.

Tang, D. T.-S. (2017). All I get is an emoji: dating on lesbian mobile phone app Butterfly. Media, Culture \& Society, 39(6), 816-832.

Teng, J. E. (1996). The construction of the" traditional Chinese woman" in the Western academy: A critical review. Signs: Journal of Women in Culture and Society, $22(1), 115-151$.

Yip, K.-S. (2006). Psychology of gender identity: An international perspective. Nova Science Publishers. 